\title{
Wirelessly Interfacing Objects and Subjects of Healthcare System - IoT Approach
}

\author{
Damian Dziak $^{1}$, Bartosz Jachimczyk ${ }^{1}$, Wlodek J. Kulesza ${ }^{2}$ \\ ${ }^{I}$ Faculty of Electrical and Control Engineering, Gdansk University of Technology, \\ Gabriela Narutowicza 11/12, 80-233, Gdansk, Poland \\ ${ }^{2}$ Department of Applied Signal Processing, Blekinge Institute of Technology, \\ 371 79, Karlskrona, Sweden \\ damian.dziak@pg.gda.pl
}

\begin{abstract}
Wireless sensor networks, WSN, for which development has begun by military applications, are nowadays applied to all human activities; e.g. in medicine for patience monitoring or to reduce the effects of disasters. Therefore, the WSNs area has been also one of the emerging and fast growing scientific fields. Increasing interest of WSNs is even caused by equally intense growth of interest in the Internet of Things domain, IoT, in which WSNs constitute a significant part. These reasons have brought about developing low cost, lowpower and multi-function sensor nodes. However, the major fact that sensor nodes run quickly out of energy has been an issue and many energy efficient routing protocols have been proposed to solve this problem. Case study presented in this paper concern design of WSN in IoT concept from system lifetime perspective. A hierarchical routing technique, which shows energy efficiency, has been validated. Simulation results show that chosen technique prolongs the lifetime of the WSN compared to other investigated clustering schemes. The advantages of this method are validated by comparative studies.
\end{abstract}

Index Terms - Energy efficiency; Internet of Things, routing protocol; wireless sensor networks

\section{INTRODUCTION}

World Health Organization research [1] shows that over the last 25 years life expectancy was extended significantly, e.g. in Poland life lengthened by six years from 71 to 77 years. However the life expectancy between man and women differs eight years, what causes that elder population lives alone. Some of those people have mobility difficulties, symptoms of dementia or other health problems. In such cases there is a need to increase their safety by using monitoring systems, which could e.g. help them to take medicines in time, or in a case of fall, inform appropriate services and caregivers. Therefore, modern technology provides a variety of solutions among them there is a new promising approach concerning Internet of Things, IoT. This paradigm employs among others Wireless Sensor Networks, WSN, the technology, which is essential for this paper approach.

The IoT concept [2] integrates many different physical and virtual devices, such as distributed sensor nodes, actuators, mobiles and other devices called things [3], [4]. It has to be emphasised that WSN constitutes a significant part

Manuscript received 11 September, 2015; accepted 4 February, 2016. of IoT [5], [6] because this technology is feasible in many areas of everyday life, industry and science, including infrastructure protection [3], diagnostics, industrial measurements, and also environmental and health monitoring [7]-[9].

Figure 1 illustrates how healthcare applications benefit from combining WSN technology into IoT paradigm. The WSN consists of network nodes where each node assembles and processes information from the environment and then sends it directly or indirectly to the base station and then to Internet. Such solutions could be used e.g. for tracking or monitoring vital signs of patients.

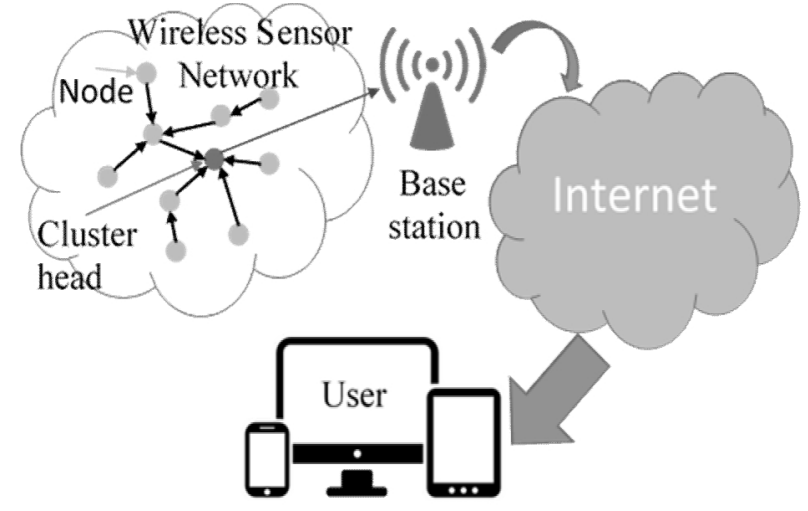

Fig. 1. Example integration of WSN in IoT.

Most of nodes constituting WSN in IoT applications are battery-powered, then the energy issue becomes one of constraints limiting the whole network lifetime and thereby its applicability for healthcare purposes. Since sending information from a node requires most of energy, the way in which information is sent within the network and its quantity are essential for system lifetime [10].

Moreover, while designing a WSN based healthcare system in IoT paradigm, it is necessary to consider organization and structure of the network, which also affect its lifetime. The structure in turn determines the type of possible transmission protocols, which depends on the node location method and the required security [11].

The advantages of WSN combined within IoT paradigm facilitates design of an energy efficient healthcare system for patient monitoring. The paper focuses on the design process of a multi-sensor system for patient monitoring based on 
WSN in IoT paradigm, and considers the various aspects of the design, from the healthcare perspective. A case study of design of Wireless Body Area Network, WBAN, including an aspect of system lifetime in the design process is presented.

\section{RELATED WORK}

The advantages and variety of wireless sensor networks make this technology suitable for IoT application in healthcare sensing systems. Among others the adaptability of smart wireless sensor node, which can adjust both its internal and external conditions is the crucial advantage. Due to this the entire network is scalable, reconfigurable and capable to auto-adapt in a case when some of the network components fail or degrade, or if networks' tasks or requirements change [5].

The driver of wide range of applications of WSN in IoT domain is development and technological capability of Micro-Electro-Mechanical Systems, MEMS, which constitute WSN sensors. Through this technology the miniaturized, high performance sensors have become cheaper and therefore widely available and commonly used [12], [13].

W.-Y. Chung, S.-C. Lee, and S.-H. Toh in [14] use advantages of wireless electrocardiography, ECG, and blood pressure sensors and combine them with cellular phone sending information to a hospital server when any suspected or unknown pattern of signals is detected. G. Bakul, D. Singh, and D. Kim [15] apply wireless Ultra-Wide Band, UWB, in an ECG monitoring system, which can be used at the same time by many patients in hospitals or other healthcare facilities. Data are gathered by wireless nodes, sent to an access point and then upload on a remote server via Internet. For wireless oxygen saturation and heart rate monitoring C. Rotariu and V. Manta [16] use Micro Power Oximeter Board from Smiths Medical and Texas Instruments module.

Many healthcare applications require to know actual position of either patient or equipment. A popular indoor localization approach is based on receiving signal strength, RSS, measurement. In this methods the localization is performed based on strength of Wi-Fi [17] or RFID [18] radio signal. A. Dobîrcău, et al. [19] in order to fit their system to IoT paradigm use low powered Wi-Fi RFID active devices, which send gathered information to an access point coupled to Internet. An interesting concept presented by Matteo Faraone, et al. allows to localize patient inside a room without a need to wear any device just by analysing RSS disturbance between sensor rows, evenly distributed on each wall of the room [20].

The localization methods for indoor purposes frequently are based on accelerometer, compass and gyroscope data e.g. Pedestrian Dead Reckoning method, PDR [21]. Superiority of these solutions is because they allow not only to localize a patient in a building with accuracy about one meter, but also the same data can be used for other purposes such as tumble detection [22] and even posture recognition [23].

Though in the most healthcare applications, just indoor localization is sufficient, sometime there is a need to track a person outdoor. In such a case wireless sensor nodes are equipped with a Global Positioning System, GPS, module. Today's GPS assures localization accuracy in city environment of about $6 \mathrm{~m}$ [24]. However, Chenshu Wu at al. show that by combination of GPS with PDR based on accelerometer, it is possible to increase localization accuracy up to $4 \mathrm{~m}$.

Advantages and diversity of wireless sensor nodes and their applicability in IoT paradigm justify healthcare related applications called Wireless Body Area Networks, WBAN. Using several different sensor nodes, S.-L. Tan, J. GarciaGuzman, and F. Villa-Lopez designed system for body temperature, heart beat rate, blood oxygen saturation and blood pressure measurements which using Wi-Fi transmits gathered data to a base station [25]. Moreover, they use accelerometers for posture and falls detection.

J. Wannenburg and R. Malekianc also developed the mobile health device for monitoring blood pressure, heart rate, oxygen saturation and skin temperature [26].They apply Bluetooth technology to transmit data to a smartphone.

Capabilities of WSN based IoT systems enable comprehensive recognition of patient behaviour. Systems, recognizing patient behaviour and classifying it as typical, abnormal or dangerous are helpful for patients at first stages of dementia or elderly people living alone, and even in caregiving facilities. L. Sun et al. use mobile phone with embedded accelerometer and Support Vector Machine, SVM based classifier to recognize different activities like walking, running, bicycling and others [27]. Liang Wang, et al. [28] designed a WBAN consisting of accelerometers and coin size RFID readers mounted on each hand of supervised people, and one additional accelerometer mounted on their waist. Furthermore, they apply passive RFID tags for labelling the equipment related to patient's different activities e.g. iron, mug, computer, etc. Using such system they recognize 25 different activities by matching current accelerometer data with the pre-defined activity set and identified RFID tags. M. Henar, et al. [29] use a Google Nexus $\mathrm{S}$ accelerometer, gyroscope, magnetometer, light and proximity sensor applied to fuzzy classifier to recognise body position and other activities.

Clifton Phua et al. [30], gather information from pressure, infrared and video sensors, accelerometer, RFID tags and compare them with 2-layer erroneous-plan recognition system which classifies behaviours based on blacklist, whitelist and naïve Bayesian classifier. The disadvantage of this method is that most of sensors do not operate wirelessly.

Another important aspect of WSN based IoT monitoring systems is patient's privacy. Most solutions using WSN in IoT domain do not apply a global addressing scheme [31]. Data gathered by an ad hoc established network are aggregated inside the network and then sent by cluster heads to the base station, which has its own IP address and is able to transmit information via Internet to the final user. Such solutions however can suffer of data security problem, due to vulnerability on attacks like: sinkhole attack, sniffing or energy drain [32]. Authors of [33] and [34] propose secure communication in WSN based on pairing identity encryption and pairing based cryptography over an elliptic curve respectively. Nevertheless, scalability of WSN using such 
data security methods is limited due to required additional power supplies for cluster heads.

Secure solutions for easy scalable networks was proposed by Md Mizanur Rahman et al. for WSN using identity-based encryption and pairing based cryptography [35]. For WBAN, Rghioui, L'aarje, Elouaai and Bouhorma [36], and Li, Lou and Ren [37] apply symmetric cryptography with a session key management system and a node authentication model with a special ID and attribute-based encryption access control respectively.

As it was mentioned, considering a WSN for healthcare application in IoT domain it is necessary to concern energy issue. As the transceiver consumes the most power of wireless sensor [38], an adequate communication technique has to ensure the energy conservation. This could be obtained with clustering technique [39]. The amount of consumed energy depends on the cluster size, smaller clusters consume less energy for internal communication but such a solution requires more energy for the external communication. Therefore, for this approach it is necessary to find out a trade-off between the cluster size and a number of clusters in the network [40]. Bandyopadhyay and Coyle [41] apply $K$-means cluster tree framework, in which the $K$ value is optimal from the point of view of energy needed for communication within a cluster. The parameter $K$ determines the maximum number of hops needed that information from any network node can reach the base station. The parameter also determines the maximum delay needed to send the data to the base station.

\section{WSN BASED IOT - PERSPECTIVE OF HEALTHCARE APPLICATION}

As the survey of related work shows, a number of nowadays solutions of IoT sensing systems is based on WSN and many of them are designed for healthcare purposes. Due to system complexity, the design process of such system requires a comprehensive approach. Figure 2 shows a flow of design process and interrelationships among different features and actors of the process, which are described in the following sections.

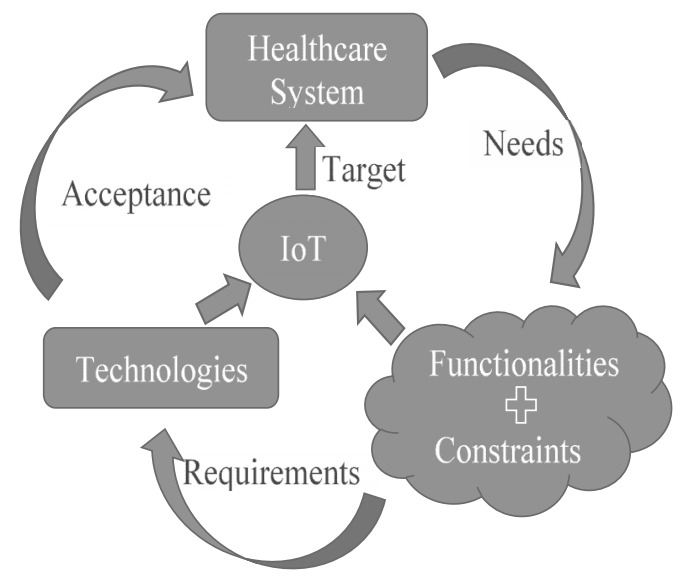

Fig. 2. Healthcare application features and relationship among them in system design process.

\section{A. Healthcare System Target}

The system contractor and/or service provider define the target and related needs, which define system functionalities and constraints. This way, the stockholders indirectly affect system final structure. A precisely defined system target is essential since systems even in the same application domain e.g. healthcare, could differ in many ways. The target, functionalities and system constrains together constitute design requirements.

This stage of the design is crucial for the final solution and requires cooperation of multidisciplinary team consisting of medical experts and investors, but even the future users who may have their wishes and limitations.

\section{B. Functionalities and Constrains}

An example of the system constrains expected from healthcare application is user's comfort. MEMS based WSN nodes without compromising prices and social aspects of the product, can satisfy comfort requirements and provide sensors that can be placed on patients, monitor their life parameters, tracking movement, analyse behaviour and even call for help while life threatening situations.

One of crucial functionalities of healthcare application is monitoring of patient's vital signs. Survey of related works indicates that wireless sensors facilitate monitoring among others ECG, blood pressure, oxygen saturation, and body temperature.

Features, which are especially valuable in healthcare applications with mobile patients, are their localization and movement tracking. In IoT domain, Wi-Fi or RFID technologies are most convenient for indoor localisation and tracking. Other useful technologies are accelerometers, compasses and gyroscopes, which not only allow patient's localization, but could be also used for posture recognition or tumble detection. When patients are allowed to stay outdoors, then localization data are supported by GPS.

In a case of serious diseases such as dementia the healthcare application may require analysing patient's behaviour or its changes, and reporting caregivers when current behaviour deviates from standard one.

Since most of wireless sensor nodes use batteries, system lifetime demands techniques saving energy and it is an important system constrains, which each designer of IoT healthcare application has to focus on. Discrete measurement methods belong to the energy saving solutions because the sensors are active just for short sampling instants. However, this sampling strategy is applicable just for slowly changing phenomena e.g. temperature or humidity. Detection of instantaneous, rapidly changing phenomena such as an acoustic pulse is considerably more complex. Another problem of measuring unsteady phenomena can be solved by means of its rarely sampling in node's sleep mode and awakening at an instant when a specific event occurs [12], [42].

WSN lifetime is determined by the vigilance coefficient, which is the ratio of the sensor's active time to its sleep time and the probability that the sensor is chosen as a cluster head or as a normal node. If $P(c)$ is the probability that the sensor is a cluster head and $P(n)$ denotes the probability that the sensor is an ordinary node in an active state then the current consumed by a sensor node can be estimated by [13]

$$
I_{c}=P(c) \times I_{a}+(1-P(c)) \times\left(P(n) \times I_{a}+(1-P(n)) \times I_{S}\right),
$$


where $I_{a}$ and $I_{s}$ are the maximum currents (in $\mathrm{mA}$ ) of sensors in active and asleep states respectively, assuming that there is no other activity in the sensor node. Then the sensor's lifetime can be calculated using (1).

The lifetime constrain can be also approached from perspective of optimal design of WBAN, by studying the joint data routing and relay positioning problem [43].

Another constrain expected from good healthcare applications is preservation of patient privacy, understood as personal privacy as well also as collected data security. Wireless sensor networks are suited for both of these cases.

\section{Technologies of WSN Healthcare Application in IoT}

Presented functionalities and constraints defined by the system target together with IoT constraints constitute requirements for technologies, which could be used. For example, the healthcare target of designed system together with requests of WSN in IoT domain, constitute requirements for applicable technologies considering only wireless communication standards [30] like Wi-Fi, ZigBee [44], Bluetooth Low Energy, BLE, [45] or RFID [46].

Concerning energy limitations along with restraints of processing and storage capabilities of WSN in IoT domain, the functionalities and requirements of designed system determine a framework of routing methods and algorithms. The main types of routing algorithms are:

- data-oriented e.g. Sensor Protocol for Information via Negotiation, SPIN [47],

- hierarchical e.g. Low Energy Adaptive Clustering Hierarchy, LEACH [48],

- location-based e.g. Minimum Energy Communication Network, MECN, [49],

- based on Particle Swarm Optimization technique [50],

- Multi-Criteria Decision Analysis and Entropy Weights, EMCR, [51].

To assure an efficient and reliable transmission from network nodes to the base station and then via Internet to final user, the network should be properly organized. Clustering based on nodes' neighbourhood is one of the effective hierarchical sensor network organizations. In this method, each node, after identifying a base station, transmits data via head nodes of intermediate clusters to the base station. The method assures bandwidth and energy efficiencies by decreasing transmissions between clusters, optimizing transmission path and data fusion, which is in line with the system requirements and functionalities.

The conducted survey reveals several basic techniques of cluster forming e.g. Random Competition based Clustering, RCC [52], which applies a random timer. The registration of nodes to each cluster is based on a rule called First Declaration Wins, FDW [53]. According to the rule, the governing function is assigned to the node, which the first declares to become a cluster head.

Another important issue of cluster formation is a transmission method. There are two possible transmission methods: direct- and multi-hop-broadcasting, [39]. In a network with the direct transmission, a request initiating a cluster is randomly sent by a node. Direct transmission method is easy to implement but is not energy efficient, since it requires sending information to all network nodes.
Even nodes located far away from the head node receive a call with no chance of its acceptance. In multi-hop transmission there are specified cluster ranges where the nodes are invited to participate in. The disadvantage of this method is a significant transmission delay compared to the direct technique. The delay is caused by data processing at each of the nodes along the multi-hop transmission path.

When the cluster is formed the selected head node governs the cluster. This node is responsible for data aggregation and control of transmission algorithms from each cluster node to the base station. In the case of a cluster with many nodes, load of the head node is greater in order to properly receive, aggregate and re-transmit the data. Since the role of the cluster head requires much energy, hence the rotation of the cluster head can lengthen the cluster life.

The cluster head can be chosen randomly or pre-defined by the network designer. Random assignment of the heading functions is based on the probability that the new selected node has never been the cluster head. This rotating assignment leads to reduction of the load on the heading node.

If selection of the cluster head is based on a power residuum, then the heading role is taken by a node having the most energy. The roles can change after the end of the cycle when it is noticed that heading node energy falls below the average energy of all clusters. This method of selecting the heading node significantly lengthens the network life [54].

Another way of allocation of cluster heading function is based on the principle of minimizing the total distance from the cluster head to all nodes in the cluster. Since the transmission energy depends on the distance to the receiver the alternative minimum transmission path reduces power consumption in the cluster [55]. Hamed extended this approach with unequal size clustering formation, where clusters closer to the base station, which have to re-transmit data even from farther clusters, have fewer nodes than clusters far from base station [56].

Further constrain for WSN healthcare application in IoT paradigm is a hardware matching. In a case when sensors are worn by a human, the proposed device cannot cause any discomfort and should be as light as possible and of compact size. They should also be compatible with chosen communication technologies and allow adding extra sensors and/or remove these unnecessary. Such capabilities, are provided, inter alia by an Arduino platform [57].

The presented analysis justifies the use of IoT technologies for healthcare application, however, the design process should be a well-planned, because beside the target there must also be considered functionalities and constrains which constitute the important requirements of designed system.

\section{A Case Study of Design a Multi-Sensor HEALTHCARE APPLICATION IN IOT PARADIGM}

Considering the target of design application as a patient monitoring in nursing home care and defined earlier requirements and technologies, some design aspects need to be considered and verified as it is exemplified in this section. 
The case study presented in this chapter consists of two approaches. Section $A$ concerns design of WBAN dedicated for nursing home care patient monitoring. The second approach deals with energy efficient root node selection [58] and cluster formation method [59], [60] presented in sections $B$ and $C$ respectively.

\section{A. Design of Arduino based Wireless Body Area Network}

One of possible technologies matching mentioned targets is Arduino solution, a commonly available electronics prototyping platform, which is inexpensive, open-source, easy programing with availability of many extensions, actuators and sensors.

An Arduino board, equipped with modules such as AltIMU-10 V4, GPS/GPRS/GSM V3.0, Polar T34 Heart Rate monitor and WiDo Wi-Fi IoT Node, is designed and prototyped allowing monitoring patients in nursing home care, see Fig. 3.

AltIMU-10 v4 module with gyroscope, accelerometer and magnetometer and using PDR method are applied to localize a patient inside the nursing home care building. Moreover, accelerometer and altimeter data are used for tumble detection and posture recognition. In a case of localization outside the building GPS/GPRS/GSM V3.0 module is employed.

Data form Polar T34 heart rate monitor are exploited as additional assessment supporting decision if abnormal situations like a tumble actually occurred, because during such situation heart pulse of monitored person is higher than in normal position. Moreover, the system reports to caregivers if low or high heart rate has lasted for a longer time.

WiDo WIFI IoT node operates for communication with the base station, which aggregates and sends data via Internet to the caregivers in nursing home care. Wi-Fi standard is used to facilitate internal communication among other nodes, constituting WSN.

Mentioned modules are designed to be mounted on a chest belt and are managed with the Arduino Leonardo board.

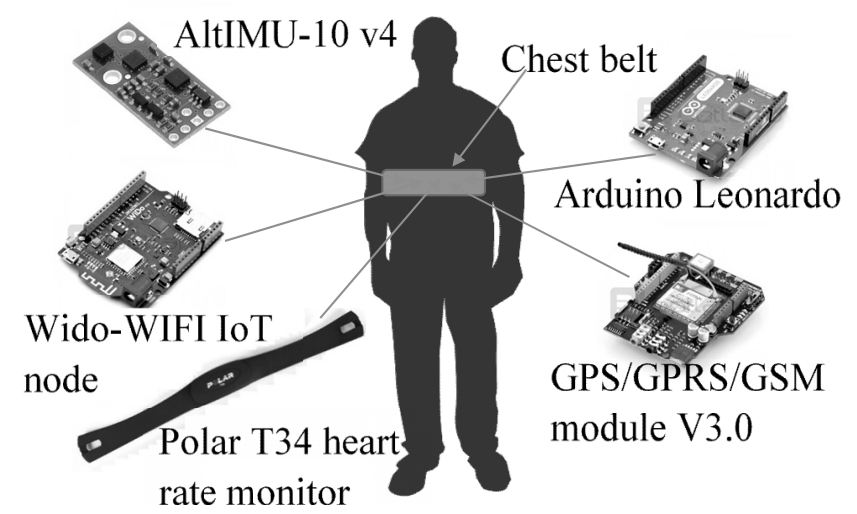

Fig. 3. Components of the proposed WBAN system for patient monitoring in nursing home care.

\section{B. Modified Algorithm of a Root Node Selection}

As the IoT paradigm requires reliable access to things, then to assure the system reliability, its lifetime becomes the crucial system parameter.
In the proposed modification of the LEACH routing protocol, the choice of the cluster head is based on prediction of energy consumption in the next transmission round and minimizing the data transmission path to the base station. The proposed algorithm consists of four stages:

- dynamic formation of clusters based on the geographic principle,

- selection of all cluster heads,

- in each cluster, aggregation of data from all cluster nodes in the cluster head,

- data transmission to the base station or to the cluster head located closer to the base station.

The procedure of selecting the cluster heads starts from a clusters closest to the base station. Initial energy $E_{i n}$, of each cluster node and its distance $d$ to the base station are estimated. Then the energy required for transmission $k$ bytes from any potential cluster head to the base station is calculated using the relationship: $E_{a m p} \times k \times d^{2}$ and then based on the relationship: $E_{i n}-E_{a m p} \times k \times d^{2}$, a node which has the largest energy residuum is appointed to be the cluster head. The same procedure is repeated successively to the next clusters further from the base station using estimated distance $d$ to the head of the cluster closer to the base station.

The procedure was implemented in Matlab and simulated using parameters listed in Table I.

TABLE I. SIMULATION PARAMETERS.

\begin{tabular}{|c|c|c|c|}
\hline Parameters & Symbol & Unit & Value \\
\hline Number of nodes & $N$ & - & 250 \\
\hline Initial energy of each node & $E_{\text {in }}$ & $\mathrm{J}$ & 200 \\
\hline Transmission packet size & $K$ & byte & 100 \\
\hline $\begin{array}{c}\text { Energy consumption per bit to } \\
\text { run the transmitter }\end{array}$ & Eelect & $\mathrm{nJ} / \mathrm{byte}$ & 50 \\
\hline $\begin{array}{c}\text { Energy consumption per bit to } \\
\text { send signal into the open space }\end{array}$ & Eamp & $\mathrm{pJ} / \mathrm{bit}$ & 100 \\
\hline Network area & $S$ & $\mathrm{~m} * \mathrm{~m}$ & $300 * 300$ \\
\hline Coordinates of the base station & $x, y$ & - & $(0,0)$ \\
\hline
\end{tabular}

250 points are randomly distributed in three equal clusters of the area of $300 \mathrm{~m} \times 300 \mathrm{~m}$, corresponding to possible positions of monitored patients in a garden what is illustrated in Fig. 4. Lifetime curves of the proposed system for three different network structures: non-hierarchical, and hierarchical of first- and second-order are shown in Fig. 5.

The nodes lifetime curves prove the advantage of hierarchical structures. The death of first node in the first level hierarchical case is observed ten times later than in the non-hierarchical case and the system is able to work almost 1.5 times longer.

Moreover, Fig. 6 shows histogram of the residual energy after 300 rounds for non-hierarchical and hierarchical of first and second-order structures. As one can see the amount of residual energy remaining in the network with nonhierarchical structure is much smaller than both first- and second-order hierarchical cases, what means much longer operation time of hierarchical network.

It could be also observed that the higher hierarchy level and a greater amount of clusters improve the network lifetime. However, it can be foreseen that the number of clusters and their populations have to match each other to optimise the network lifetime. 


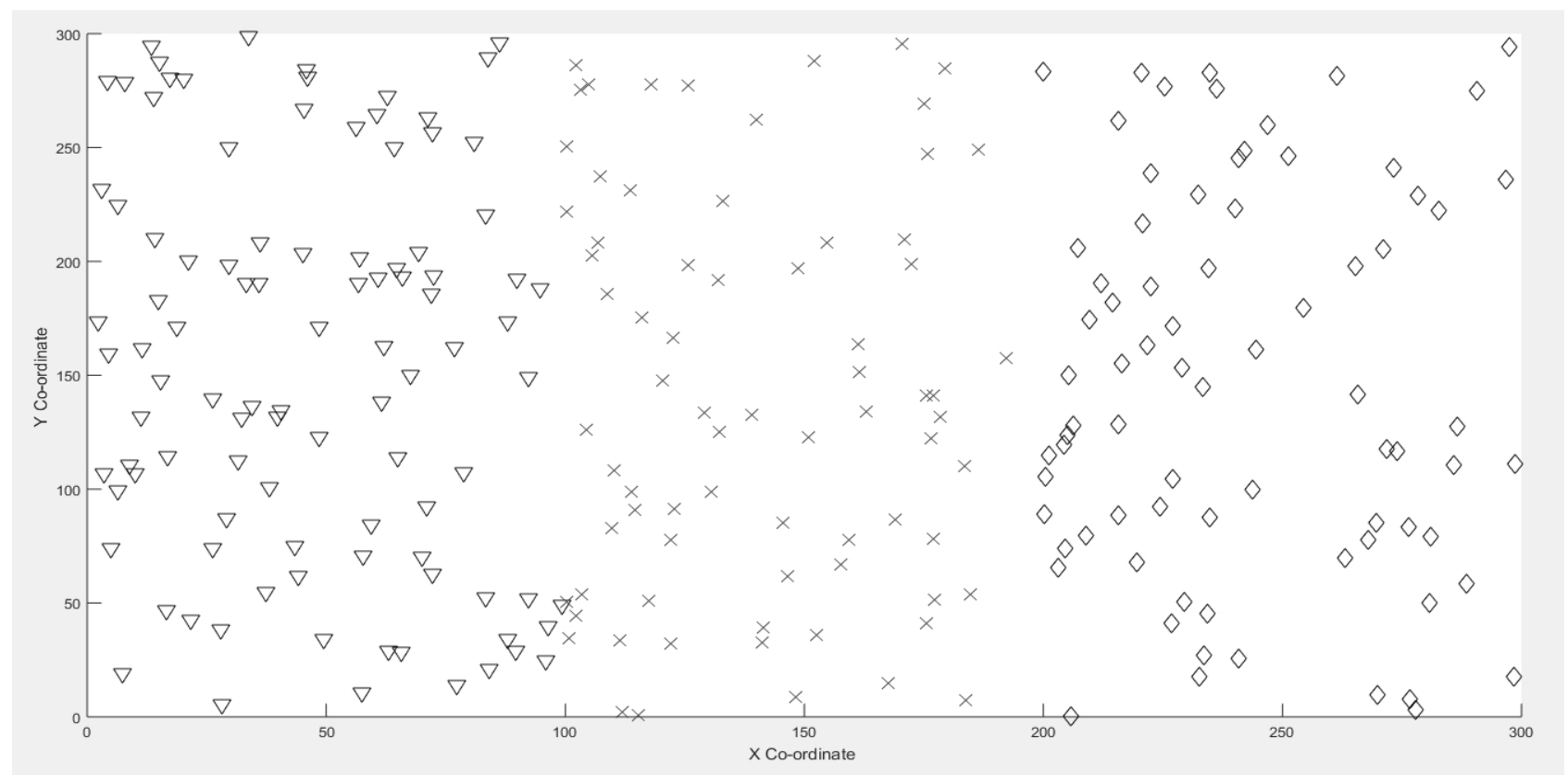

Fig. 4. Second level hierarchical formation with differentiated shape indicating the three clusters where membership to each cluster is notified by different symbol.

\section{Energy Efficient Cluster Formation Method}

Further extension of the WSN lifetime is possible due to appropriate cluster formation method. We propose using the $K$-mean algorithm to define the optimal distance between network nodes and the cluster head [61]. The proposed algorithm consists of six steps:

1. Defining a number of clusters,

2. Selecting an initial cluster headings in each cluster,

3. Estimating the distance from each node to all initial cluster headings,

4. Assigning each node to the nearest heading initiator,

5. Formation of clusters consisting of nodes placed closest to the heading initiators.

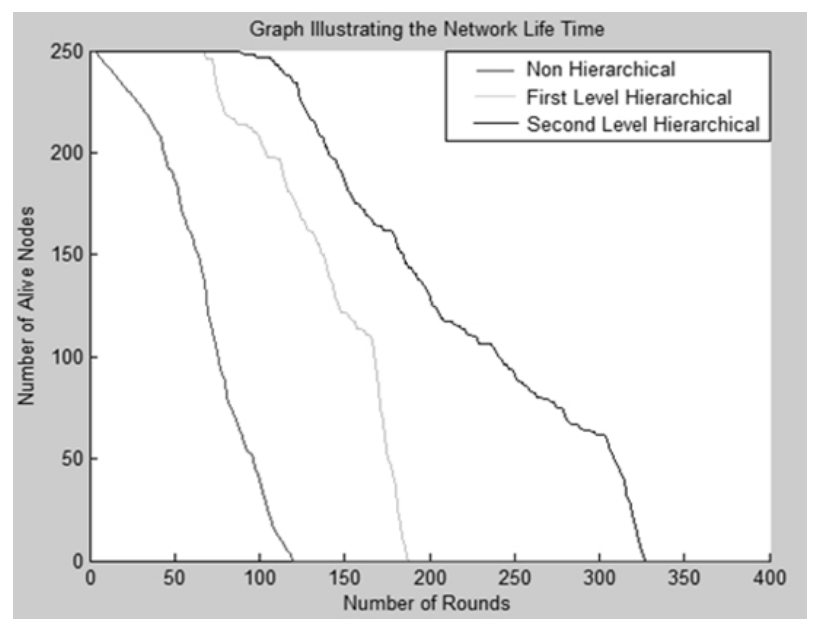

Fig. 5. Comparison of network lifetime for different level hierarchical formations applying the proposed modification of the LEACH routing protocol.

To analyse the proposed solution a case of nursing care home has been implemented and simulated in Matlab. 100 patients are randomly positioned in a garden of area $90 \mathrm{~m} \times 80 \mathrm{~m}$. Each patient is equipped with a node, with the same initial energy. The simulation results are shown in Fig. 7.
The simulation results are compared with the results of the method when clusters are formed geographically. It could be denoted that even with a small number of nodes the death of first node with $K$-mean clustering method occurred six times later than in case where clusters were formed geographically. Moreover, the proposed system was able to work almost two times longer.

\section{D.Results Discussion}

The designed, prototyped and tested system consisting of an Arduino board, equipped with modules such as AltIMU10 V4, GPS/GPRS/GSM V3.0, Polar T34 Heart Rate monitor and WiDo Wi-Fi IoT Node, is a suitable solution allowing monitoring patients in nursing home care.

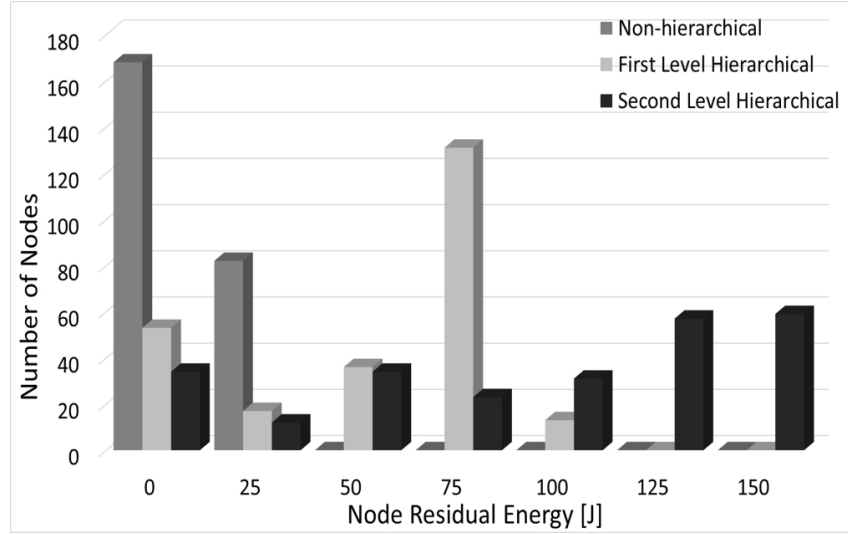

Fig. 6. Histogram of residual energy for different level hierarchical formations after 300 rounds.

Simulations results prove that modification of the LEACH routing protocol causes that death of first node occurs ten times later than in basic method and the system lifetime becomes $50 \%$ longer.

Moreover, residual energy after 300 rounds for secondorder hierarchical structure surpasses the one with nonhierarchical structure, confirming longer operation time and superiority of hierarchical structure. 


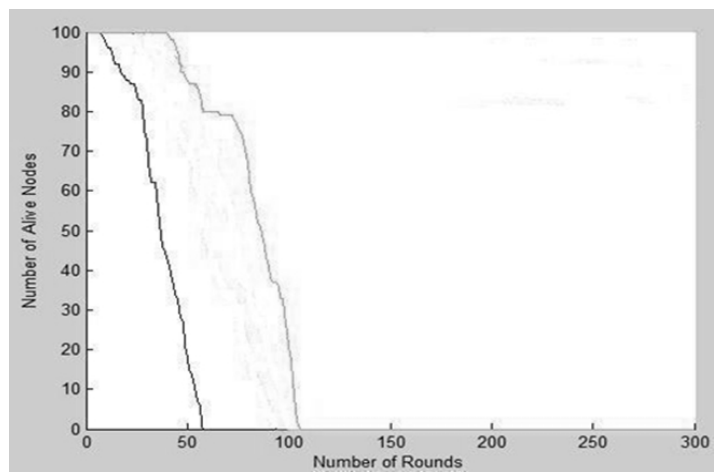

Fig. 7. Comparison of node lifetime for second level hierarchical formations and a different clustering method, the black line indicates geographical clustering and the grey one indicates clustering using K-mean method.

Furthermore, $K$-mean cluster formation method results in death of first node six times later and prolongs almost twice the system lifetime compare to geographical method.

\section{V.CONCLUSIONS}

The authors analyse the system design procedure from perspective of healthcare WSN in IoT domain. The analysed design procedure shows complexity and interdependency of the comprehensive design process. The procedure requires combined effort of many actors approaching the problem from different perspective. Precise pre-defined functionalities and constraints are essential to optimise choice of applied technologies.

The presented case study concerns a solution for nursing home care dedicated to patient supervision by means of their localization using Arduino technology equipped with accelerometer, magnetometer, health rate monitor, Wi-Fi and GPS modules.

The case study illustrates how to modify the applied methods and technologies to meet to system constraints. For instance the lifetime of analysed system can be extended by choice of the suitable energy efficient algorithm of root node selection and cluster formation method.

Future works consider implementation of the design procedure in real world. The design implementation is going to be realised as an EU project involving several contributes including institutions of public conveniences, industry and academia. Furthermore the analysis of security issues in the proposed system is planned. Moreover, the system could be extended with additional sensors useful for healthcare applications.

\section{ACKNOWLEDGMENT}

The authors wish to express their sincere thanks to Professor Zygmunt Kusmierek from the Technical University of Lodz for his support. This article is a result of authors' cooperation with Communication Research Laboratory AB Kalmar, Sweden, and Ismail Eleburuike, Soete Adekunle, Xin Qu and Sheima Hamed from Blekinge Institute of Technology.

\section{REFERENCES}

[1] "Global Health Observatory Data Repository, WHO. [Online]. Available: http://apps.who.int/gho/data/node.main.688.

[2] “Auto-ID Labs." [Online]. Available: http://autoidlabs.org/ wordpress_website
[3] L. Buttyan, D. Gessner, A. Hessler, P. Langendoerfer, "Application of wireless sensor networks in critical infrastructure protection: challenges and design options", IEEE Wirel. Commun., vol. 17, no. 5, pp. 44-49, 2010. [Online]. Available: http://dx.doi.org/10.1109/ MWC.2010.5601957

[4] L. Coetzee, J. Eksteen, "The Internet of Things - promise for the future? An introduction", in IST-Africa Conf. Proc., 2011, pp. 1-9.

[5] L. Mainetti, L. Patrono, A. Vilei, "Evolution of wireless sensor networks towards the Internet of Things: A survey", in 19th Int. Conf. Software, Telecommunications and Computer Networks, 2011, pp. 1-6.

[6] N. Khalil, M. R. Abid, D. Benhaddou, M. Gerndt, "Wireless sensors networks for Internet of Things", in IEEE Ninth Int. Conf. Intelligent Sensors, Sensor Networks and Information Processing, 2014, pp. 16. [Online]. Available: http://dx.doi.org/10.1109/issnip.2014. 6827681

[7] A. A. Kumar, S. K. Ovsthus, L. M. Kristensen, "An industrial perspective on wireless sensor networks - a survey of requirements, protocols, and challenges", IEEE Commun. Surv. Tutor., vol. 16, no. 3, pp. 1391-1412, 2014. [Online]. Available: http://dx.doi.org/ 10.1109/SURV.2014.012114.00058

[8] A. de la Piedra, F. Benitez-Capistros, F. Dominguez, A. Touhafi, "Wireless sensor networks for environmental research: A survey on limitations and challenges", 2013, pp. 267-274.

[9] M. Balouchestani, K. Raahemifar, S. Krishnan, "Wireless Body Area Networks with compressed sensing theory", in ICME Int. Conf. Complex Medical Engineering, 2012, pp. 364-369. [Online]. Available: http://dx.doi.org/10.1109/iccme.2012.6275663

[10] N. Pantazis, S. Nikolidakis, D. D. Vergados, "Energy-efficient routing protocols in wireless sensor networks: a survey", IEEE Commun. Surv. Tutor., vol. 15, no. 2, pp. 551-591, 2013. [Online]. Available: http://dx.doi.org/10.1109/SURV.2012.062612.00084

[11] M. Sain, P. Kumar, H. J. Lee, "A survey of middleware and security approaches for Wireless Sensor Networks", in 6th Int. Conf. Computer Sciences and Convergence Information Technology, 2011, pp. 64-69.

[12] C. Pang, M. Yu, X. M. Zhang, A. Gupta, K. M. Bryden, "Multifunctional optical MEMS sensor platform for wireless optical sensor networks", in 16th Int. Conf. Solid-State Sensors, Actuators and Microsystems, 2011, pp. 1566-1569. [Online]. Available: http://dx.doi.org/10.1109/TRANSDUCERS.2011.5969739

[13] D. Ruffieux, M. Contaldo, C. Enz, "MEMS-based all-digital frequency synthesis for ultralow-power radio for WBAN and WSN applications", in IEEE Int. Symposium on Circuits and Systems, 2011, pp. 157-160. [Online]. Available: http://dx.doi.org/ 10.1109 /iscas. 2011.5937525

[14] W.-Y. Chung, S.-C. Lee, S.-H. Toh, "WSN based mobile u-healthcare system with ECG, blood pressure measurement function", in 30th Annual Int. Conf. IEEE Engineering in Medicine and Biology Society, 2008, pp. 1533-1536.

[15] G. Bakul, D. Singh, D. Kim, "Optimized WSN for ECG monitoring in ubiquitous healthcare system", in 4th Int. Conf. Interaction Sciences, 2011, pp. 23-26.

[16] C. Rotariu, V. Manta, "Wireless system for remote monitoring of oxygen saturation and heart rate", in Federated Conf. Computer Science and Information Systems, 2012, pp. 193-196.

[17] P. Bahl, V. N. Padmanabhan, "RADAR: an in-building RF-based user location and tracking system", in Proc. Nineteenth Annual Joint Conf. IEEE Computer and Communications Societies, 2000, vol. 2, pp. 775-784. [Online]. Available: http://dx.doi.org/10.1109/infcom. 2000.832252

[18] B. S. Jachimczyk, D. M. Dziak, W. J. Kulesza, "Performance analysis of an RFID-based 3D indoor positioning system combining scene analysis and neural network methods", in Scientific Papers of Faculty of Electrical and Control Engineering Gdansk University of Technology, 2013, vol. no. 34, pp. 29-33.

[19] A. Dobircau, S. Folea, H. Valean, D. Bordencea, "Indoor localization system based on low power Wi-Fi technology", in $19^{\text {th }}$ Telecommunications Forum, 2011, pp. 317-320. [Online]. Available: http://dx.doi.org/10.1109/telfor.2011.6143553

[20] M. Faraone, R. Alesii, S. Tennina, F. Graziosi, "Device free patients localization in controlled indoor environments", in 2014 IEEE 19th Int. Workshop on Computer Aided Modelling and Design of Communication Links and Networks, 2014, pp. 149-153. [Online]. Available: http://dx.doi.org/10.1109/camad.2014.7033224

[21] H.-H. Hsu, W.-J. Peng, T. K. Shih, T.-W. Pai, K. L. Man, "Smartphone indoor localization with accelerometer and gyroscope", in 17th Int. Conf. Network-Based Information Systems, 2014, 
pp. 465-469. [Online]. Available: http://dx.doi.org/10.1109/nbis. 2014.72

[22] L. Xiao-qin, C. Wei-min, "The alarm system of elder tumble at the geracomium based on ZigBee", in Int. Conf. Electronics and Optoelectronics, 2011, vol. 2, pp. 38-40. [Online]. Available: http://dx.doi.org/10.1109/iceoe.2011.6013168

[23] H. Gjoreski, M. Lustrek, M. Gams, "Accelerometer placement for posture recognition and fall detection", in 7th Int. Conf. Intelligent Environments, 2011, pp. 47-54. [Online]. Available: http://dx.doi.org/10.1109/ie.2011.11

[24] C. Wu, Z. Yang, Y. Xu, Y. Zhao, Y. Liu, "Human mobility enhances global positioning accuracy for mobile phone localization", in IEEE Trans. Parallel Distrib. Syst., 2015, vol. 26, no. 1, pp. 131-141. [Online]. Available: http://dx.doi.org/10.1109/TPDS.2014.2308225

[25] S.-L. Tan, J. Garcia-Guzman, F. Villa-Lopez, "A wireless body area network for pervasive health monitoring within smart environments", in IEEE Int. Conf. Consumer Electronics, 2012, pp. 47-51. [Online] Available: http://dx.doi.org/10.1109/icce-berlin.2012.6336471

[26] J. Wannenburg, R. Malekianc, "Body sensor network for mobile health monitoring, a diagnosis and anticipating system", in IEEE Sensors Journal, vol. 15, no. 12, pp. 6839-6852, 2015. [Online] Available: http://dx.doi.org/10.1109/JSEN.2015.2464773

[27] L. Sun, D. Zhang, B. Li, B. Guo, S. Li, "Activity recognition on an accelerometer embedded mobile phone with varying positions and orientations", in Ubiquitous intelligence and computing, 2010, pp. $548-562$.

[28] L. Wang, T. Gu, H. Chen, X. Tao, J. Lu, "Real-time activity recognition in wireless body sensor networks: from simple gestures to complex activities", in 16th Int. Conf. Embedded and Real-Time Computing Systems and Applications, 2010, pp. 43-52. [Online]. Available: http://dx.doi.org/10.1109/rtcsa.2010.19

[29] H. Martin, J. Iglesias, J. Cano, A. M. Bernardos, J. R. Casar, "Towards a fuzzy-based multi-classifier selection module for activity recognition applications", in Int. Conf. on Pervasive Computing and Communications Workshops, 2012, pp. 871-876. [Online]. Available: http://dx.doi.org/10.1109/percomw.2012.6197634

[30] C. Phua, V. S.-F. Foo, J. Biswas, A. Tolstikov, A.-P.-W. Aung, J. Maniyeri, W. Huang, M.-H. That, D. Xu, A. K.-W. Chu, "2-layer Erroneous-Plan Recognition for dementia patients in smart homes", in 11th Int. Conf. e-Health Networking, Applications and Services, 2009, pp. 21-28. [Online]. Available: http://dx.doi.org/10.1109/ health.2009.5406183

[31] B. Krishnamachari, D. Estrin, S. Wicker, "The impact of data aggregation in wireless sensor networks", in 22nd Int. Conf. Distributed Computing Systems Workshops, 2002, pp. 575-578. [Online]. Available: http://dx.doi.org/10.1109/icdesw.2002.1030829

[32] S. Sundar Prasad, C. Kumar, "An energy efficient and reliable internet of things", in Int. Conf. Communication, Information Computing Technology, 2012, pp. 1-4. [Online]. Available: http://dx.doi.org/10.1109/iccict.2012.6398115

[33] M. Rahman, N. Nasser, K. Saleh, "Identity and pairing-based secure key management scheme for heterogeneous sensor networks", in Int. Conf. Wireless and Mobile Computing, Networking and Communications, 2008, pp. 423-428. [Online]. Available: http://dx.doi.org/10.1109/wimob.2008.79

[34] S. M. Mizanur Rahman, K. El-Khatib, "Private key agreement and secure communication for heterogeneous sensor networks", in $J$ Parallel Distrib. Comput., vol. 70, no. 8, pp. 858-870, 2010 [Online]. Available: http://dx.doi.org/10.1016/j.jpdc.2010.03.009

[35] S. Md Mizanur Rahman, et al., "A lightweight secure data aggregation technique for wireless sensor network", in International Symposium on Multimedia, 2014, pp. 387-392.

[36] A. Rghioui, A. L'aarje, F. Elouaai, M. Bouhorma, "The Internet of Things for healthcare monitoring: Security review and proposed solution", in Third IEEE Int. Colloquium in Information Science and Technology, 2014, pp. 384-389. [Online]. Available: http://dx.doi.org/10.1109/cist.2014.7016651

[37] M. Li, W. Lou, K. Ren, "Data security and privacy in wireless body area networks", in IEEE Wirel. Commun. vol. 17, no. 1, pp. 51-58, 2010.

[38] T. He, S. Krishnamurthy, J. A. Stankovic, T. Abdelzaher, L. Luo, R. Stoleru, T. Yan, L. Gu, "Energy-efficient surveillance system using wireless sensor networks", in In Mobisys, pp. 270-283, 2004 [Online]. Available: http://dx.doi.org/10.1145/990064.990096

[39] A. A. Abbasi, M. Younis, "A survey on clustering algorithms for wireless sensor networks", Comput. Commun., vol. 30, no. 14-15, pp. 2826-2841, 2007. [Online]. Available: http://dx.doi.org/10.1016/ j.comcom.2007.05.024
[40] D. Wei, H. Chan, "Clustering ad hoc networks: schemes and classifications", in 3rd Annual IEEE Communications Society on Sensor and Ad Hoc Communications and Networks, 2006, vol. 3, pp. 920-926.

[41] S. Bandyopadhyay, E. J. Coyle, "An energy efficient hierarchical clustering algorithm for wireless sensor networks", 2003, vol. 3, pp. 1713-1723.

[42] S. Sundar Prasad, C. Kumar, "An energy efficient and reliable internet of things", in Int. Conf. Communication, Information Computing Technology, 2012, pp. 1-4. [Online]. Available: http://dx.doi.org/10.1109/iccict.2012.6398115

[43] J. Elias, A. Mehaoua, "Energy-aware topology design for wireless body area networks", in Int. Conf. Communications, 2012, pp. 34093410. [Online]. Available: http://dx.doi.org/10.1109/icc.2012. 6363949

[44] Anurag, S. R. Moosavi, A.-M. Rahmani, T. Westerlund, G. Yang, P. Liljeberg, H. Tenhunen, "Pervasive health monitoring based on Internet of Things: Two case studies", in 4th Int. Conf. Wireless Mobile Communication and Healthcare, 2014, pp. 275-278

[45] A. E. Boualouache, O. Nouali, S. Moussaoui, A. Derder, "A BLEbased data collection system for IoT", in First Int. Conf. New Technologies of Information and Communication, 2015, pp. 1-5. [Online]. Available: http://dx.doi.org/10.1109/ntic.2015.7368748

[46] L. Catarinucci, et al., "An IoT-aware architecture for smart healthcare systems", in IEEE Internet Things $J .$, no. 99, pp. 1-4, 2015

[47] W. R. Heinzelman, A. Chandrakasan, H. Balakrishnan, "Energyefficient communication protocol for wireless microsensor networks", in Proc. 33rd Annual Hawaii Int. Conf. System Sciences, 2000, pp. 2-10. [Online]. Available: http://dx.doi.org/10.1109/HICSS.2000. 926982

[48] K. Akkaya, M. Younis, "A survey on routing protocols for wireless sensor networks", in Ad Hoc Netw., vol. 3, no. 3, pp. 325-349, 2005 [Online]. Available: http://dx.doi.org/10.1016/j.adhoc.2003.09.010

[49] J. Kulik, W. Heinzelman, "Negotiation-based protocols for disseminating information in wireless sensor networks", in Wirel. Netw., vol. 8, pp. 169-185, 2002. [Online]. Available: http://dx.doi.org/10.1023/A:1013715909417

[50] M. J. Handy, M. Haase, D. Timmermann, "Low energy adaptive clustering hierarchy with deterministic cluster-head selection", in 4th Int. Workshop on Mobile and Wireless Communications Network, 2002, pp. 368-372. [Online]. Available: http://dx.doi.org/10.1109/ MWCN.2002.1045790

[51] V. Rodoplu, T. H. Meng, "Minimum energy mobile wireless networks", vol. 17, no. 8, pp. 1333-1344, 1999.

[52] S. S. Bhunia, et al., "EMCR: routing in WSN using multi criteria decision analysis and entropy weights", in Internet and Distributed Computing Systems, 2014, pp. 325-334.

[53] S. Dai, X. Jing, L. Li, "Research and analysis on routing protocols for wireless sensor networks", in Proc. Int. Conf. Communications, Circuits and Systems, 2005, vol. 1, pp. 407-411.

[54] X. Qu, "Energy efficient wireless sensor network with modified LEACH algorithm", Master Thesis, Blekinge Institute of Technology, 2012.

[55] S. Ghiasi, A. Srivastava, X. Yang, M. Sarrafzadeh, "Optimal energy aware clustering in sensor networks", in Sensors, 2002 vol. 2, no. 7 , pp. 258-269.

[56] S. H. E. Hamed, "An energy-balancing unequal clustering algorithm for multi-hop routing in WSN", Master Thesis, Blekinge Institute of Technology, 2013.

[57] M. Banzi, Getting Started with Arduino, in O'Reilly Media Inc., 2011.

[58] I., O., Eleburuike, S., S., Adekunle, "An energy efficient protocol based on hierarchical routing technique for WSN", Master Thesis Blekinge Institute of Technology, 2010.

[59] X. Cui and Z. Liu, "BCEE: A balanced-clustering, energy-efficient hierarchical routing protocol in Wireless Sensor Networks", in IEEE Int. Conf. Network Infrastructure and Digital Content, 2009, pp. 26 30.

[60] F. Tashtarian, A. Haghighat, M. T. Honary, H. Shokrzadeh, "A new energy-efficient clustering algorithm for Wireless Sensor Networks", in 15th Int. Conf. Software, Telecommunications and Computer Networks, 2007, pp. 1-6.

[61] T. Kanungo, D. M. Mount, N. S. Netanyahu, C. D. Piatko, R Silverman, A. Wu, "An efficient k-means clustering algorithm analysis and implementation", in IEEE Trans. Pattern Anal. Mach. Intell., vol. 24, no. 7, pp. 881-892, 2002. [Online]. Available: http://dx.doi.org/10.1109/TPAMI.2002.1017616 